\title{
Neurological rehabilitation inpatients frequently receive orthotic care: A retrospective cohort study.
}

Joshua Young ${ }^{1}$, Cameron Moss².

1 - Orthotic department, Queen Mary's Hospital, St George's University Hospitals NHS Foundation Trust. 2 Wolfson Neurorehabilitation Centre, Queen Mary's Hospital, St George's University Hospitals NHS Foundation Trust.

Corresponding author: joshua.young1@nhs.net

\section{Abstract}

Aim:

Orthotic management is frequently part of the rehabilitation of various neurological conditions in adults such as stroke, peripheral neuropathies, spinal cord injuries and multiple sclerosis. Despite this, there is limited data available on the implementation of orthotic care in practice. The primary aim of this study is to establish the proportion of inpatients in a mixed neurorehabilitation cohort which receive orthotic assessment and treatment. The secondary aim was to document the overall care pathway.

Method:

Existing data was reviewed retrospectively. Data gathered included patient demographics such as age, gender and diagnosis, type of orthoses prescribed, number of orthotic treatment sessions required and outcome measures used. The paper was prepared in accordance with the STROBE statement for observational studies.

Results:

During a 6 month period, 54 patients were admitted as inpatients for neurorehabilitation. Of these $25(46 \%)$ were referred to the orthotic service for assessment, with $23(43 \%)$ receiving a lower limb orthosis and 19 (35\%) subsequently being discharged using either an ankle-foot orthosis or knee- 
ankle-foot orthosis. The conditions most commonly seen in orthotic clinic were stroke $(40 \%)$, Incomplete spinal cord injuries (28\%) and peripheral neuropathies (16\%). Significant improvements were recorded at discharge using the Functional Independence Measure (FIM) and Functional Assessment Measure (FAM). Various aspects of the orthotic care process are described.

\section{Conclusion:}

This study suggests that orthotic treatment is needed with high frequency in a mixed inpatient neurorehabilitation cohort. In terms of service planning, this data supports existing the recommendation that neurological rehabilitation centres should have good access to orthotic services.

Clinical relevance:

- Custom orthoses such as AFOs and KAFOs are frequently needed by neurological rehabilitation inpatients

- Custom orthoses may facilitate standing and walking at an earlier stage during rehabilitation

- Those responsible for neurological rehabilitation services should ensure appropriate access to orthotic services

Keywords: orthotics; orthoses; neurorehabilitation; stroke

\section{Background}

Orthotists are part of the multidisciplinary team involved in the rehabiliation of long term neurological conditions (Turner-Stokes et al, 2008). Orthotic management is commonly part of the rehabilitation of neurological conditions including stroke, peripheral neuropathies, spinal cord injuries and multiple sclerosis. Despite the established use of orthoses in adults with neurological 
conditions (AwNC), there is limited data available on the implementation of orthotic care in practice; it is unclear what proportion of AwNC are prescribed orthoses and what types of orthoses are used. This is reflected by the varying recommendations made on the use of ankle-foot orthoses (AFOs) in stroke (Bowers and Ross, 2010; Bowen et al, 2016; NICE 2013).

\section{Aim}

The primary aim of this study is to establish the proportion of inpatients in a mixed neurological rehabilitation cohort at a single centre which receive orthotic assessment and treatment. The secondary aim was to document the overall pathway including patient characteristics, nature of care provided (i.e. orthoses prescribed), functional status of patients at assessment and discharge, and whether prescribed orthoses were still being used at a) discharge and b) 3 month review.

\section{Method}

A retrospective cohort study design was used. Data was collected at the [name of centre] neurorehabilitation centre, which provides inpatient neurorehabilitation. Orthotic treatment for inpatients is provided by an on-site orthotics service. The service has 26 inpatient beds for AwNC. A weekly clinic for these inpatients is conducted by an orthotist with experience in neurology, supported by a physiotherapist from the inpatient team.

Data for patients admitted during a 6 month period in 2017 was included. Existing data was reviewed retrospectively. Data gathered included patient demographics such as age, gender and diagnosis, type of orthoses prescribed, number of orthotic treatment sessions required and outcome measures. Orthoses are described based on terminology from the International Standards Organization (ISO) such as 'ankle-foot orthosis' (AFO) and 'knee-ankle-foot orthosis' (KAFO) (ISO, 1989). Functional Independence Measure (FIM), Functional Assessment Measure (FAM) and Neurological Impairment Set data (Turner-Stokes et al, 1999) was tested for 
normality using a one sample Kolmogorov-Smirnov test and compared using a paired t-test with significance at $<0.05$.

Written confirmation was gained from the trust's research governance office confirming that the study did not require formal ethical approval. The paper was prepared with reference to the STROBE statement for observational studies (Elm et al, 2007).

\section{Results}

During the period, 54 patients were admitted as inpatients. Of these, 25/54 (46\%) were referred to an orthotist for assessment during their stay. See figure 1 for patient flow through the care pathway. Patient characteristics, diagnoses and orthotic prescriptions are indicated in table 1. 9/23 (39\%) stroke patients were referred to orthotics and $16 / 31(52 \%)$ of those with all other diagnoses.

Orthotic prescription and clinical time

Twenty three out of 25 patients referred to the orthotic clinic were provided with a lower limb orthosis (see table 1). For full details of orthotic prescriptions and prescription by diagnosis see appendix. Patients required between 1 and 5 treatment sessions (mean 3.4, SD 1.26).

Use of orthoses at discharge and review

By discharge, all 23 patients initially prescribed an orthosis or orthoses were still using the device(s). At 3 months, 1 patient was lost to follow up, 1 was deceased, 1 ceased KAFO use due to terminal illness, and 1 ceased FO use due to lack of benefit. A further patient progressed from using their KAFO to an AFO. The remaining 18 patients who were reviewed at 3 months were still using their orthoses as initially prescribed. Of the initial cohort of 54 inpatients, 23 (43\%) were discharged using a lower limb orthosis. Of the initial cohort, $3(6 \%)$ were discharged with prefabricated lower limb orthoses; 20 (37\%) were discharged with custom lower limb orthoses; 19 
(35\%) were discharged using either an AFO or KAFO. 9/23 (39\%) stroke patients were discharged with an AFO or KAFO and 10/31 (32\%) of those with all other diagnoses.

Figure 1 - Patient flow through the care pathway

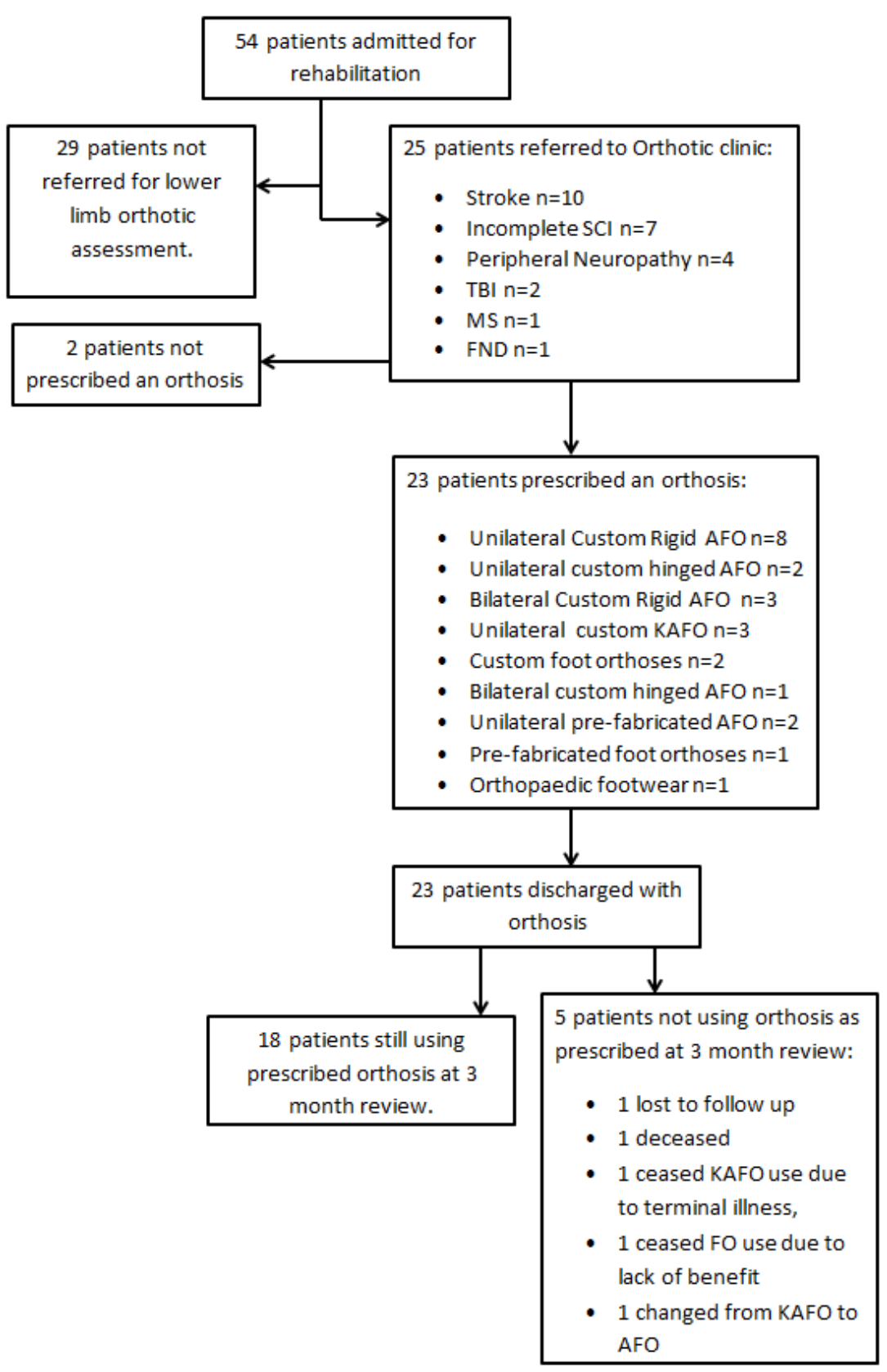

Outcome measures and functional status at assessment and discharge

During treatment within the orthotic clinic, the 10 metre walk test (10MWT) or timed up and go test (TUG) were used with $68 \%$ of patients at some stage during the treatment pathway. A numeric rating scale for pain was also used with one patient (4\%). Use of outcome measures (OMs) varied 
meaning that comparison values with and without orthoses at each stage was not always

available. At assessment in orthotic clinic, 12 patients (48\%) were recorded as being unable to complete a TUG or 10MWT without physical assistance, even if walking aids such as sticks and

Table 1 - Patient characteristics, diagnoses and orthotic prescriptions

Characteristics Number $(n=25)$, Frequency [\%]

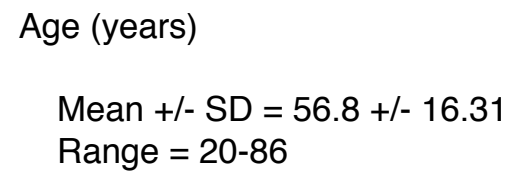

Range $=20-86$

$\begin{array}{ll}\text { Gender } & 15[60 \%] \\ & 10[40 \%] \\ \text { Male } & \\ \text { Female } & \end{array}$

Diagnosis

$\begin{array}{ll} & 10[40 \%] \\ \text { Stroke } & 7[28 \%] \\ \text { Incomplete spinal cord injury } & 4[16 \%] \\ \text { Peripheral neuropathy } & 2[8 \%] \\ \text { Brain injury } & 1[4 \%] \\ \text { Multiple sclerosis } & 1[4 \%] \\ \text { Functional neurological } & \\ \text { disorder } & \end{array}$

Orthotic prescription

Unilateral custom rigid AFO

$8[32 \%]$

Bilateral custom rigid AFOs

$3[12 \%]$

Unilateral custom KAFO

$3[12 \%]$

Custom foot orthoses

$2[8 \%]$

$2[8 \%]$

Unilateral custom hinged AFO

Unilateral pre-fabricated AFO

No prescription

$2[8 \%]$

Bilateral custom hinged AFOs $1[4 \%]$

Pre-fabricated foot orthoses $1[4 \%]$

Orthotic / orthopaedic $\quad 1[4 \%]$ footwear 
frames were allowed. $13(52 \%)$ were able to complete a TUG or 10MWT, using temporary orthoses (such a pre-fabricated AFO or soft and scotch devices) and walking aids as necessary. At fitting stage, 3 patients previously unable to complete OMs were able to complete OMs (2 completed TUG, 1 completed 10MWT) only while using their orthoses (2 KAFOs, 1 rigid AFO). At inpatient review 2-4 weeks later, a further 2 patients previously unable to complete OMs were able to complete a TUG only while using a rigid AFO. At discharge $20(87 \%)$ in the group treated with orthoses were able to walk with the use of orthoses and walking aids as necessary, although formal OMs were not used in each case, while the remaining 3 patients were using ankle foot orthoses for therapeutic standing only.

Functional Independence Measure (FIM), Functional Assessment Measure (FAM) and Neurological Impairment Set scores were assessed at admission and discharge 3 months later. Total FIM+FAM scores increased from mean 176 at admission to 223 at discharge which was significant using a paired t-test $(p=<0.0001)$. Mean Neurological Impairment Set (version 9.3) scores decreased from 16.24 at assessment to 13.13 at discharge $(p=0.0012)$.

\section{Discussion}

In this study $46 \%$ of all AwNC admitted for neurorehabilitation were referred for assessment by an orthotist during their stay, with $35 \%$ discharged using an AFO or KAFO. This suggests that orthotic treatment is needed with relatively high frequency in this mixed group. High frequency of AFO use (65\%) has previously been reported in Charcot-Marie-Tooth among adults attending an outpatient clinic (Williams et al, 2017). Studies looking at inpatient rehabilitation found that $22 \%$ (Teasell et al, 2001 ) and $30.7 \%$ (Momosaki et al, 2015) of patients with stroke were discharged with an AFO, which is lower than the $39 \%$ reported in this study. A study of lower limb orthosis use by patients with spinal cord injury found a frequency of use of $25 \%$ (Hada et al, 2018). Variables affecting referral to orthotics may include referrer's beliefs and practice, and patient presentation. Practice 
may vary in different centres; a multi-centre study or clinician survey would provide further information on practice nationally within the UK.

Considering the recorded outcome measures, it seems significant that 5 patients are recorded as being able to walk without physical assistance of clinicians only while wearing their orthoses. This effect has been reported previously (Slijper et al, 2012). This, combined with the observation that 3 subjects were discharged using their AFOs for therapeutic standing only, points to the fact that both KAFOs and rigid AFOs may be used to stabilise the knee by preventing collapse into knee flexion. This is recognised by the NICE guideline which recommends AFO use where there is ankle or knee instability or weakness (NICE, 2013). Recent work has shown that there is a need for further research in this area (McDaid et al, 2017). The significant improvements in FIM + FAM scores indicate that patients using orthoses improved during their rehabilitation. This does not isolate the effect of orthoses as other treatments, therapy and natural recovery may all be reflected by these changes in scores. Some natural recovery is suggested by the signicant change in NIS score.

As patients in the cohort were frequently discharged with custom orthoses, it seems likely that neurological rehabilitation centres should have good access to orthotic services. This is in line with existing recommendations (Bowen et al, 2016). Evidence based practice guidelines state that custom orthoses are essential in the presence of certain indications (Bowers and Ross, 2010).

Limitations of this study include the retrospective observational design and relatively small sample size. The study reflects practice in a single centre, limiting generalisability. While multiple clinicians are involved in referrals to the orthotic service and in clinical decision making, the results may be influenced by bias as a result of the authors' practice. Further work is required to define current practice concerning orthotic care provided to AwNC in other areas, in both inpatient and outpatient contexts. 


\section{Conclusion}

This brief report provides an insight into clinical practice concerning orthotic treatment in neurological rehabilitation. In this study, a large proportion of AwNC required orthotic assessment and treatment. Patients significantly improved in functional measures following rehabilitation including the use of orthoses. While practice and access to orthotic services may vary around the UK, these results imply that orthotic treatment is needed with relatively high frequency in a mixed inpatient neurological rehabilitation cohort. Further research is needed to determine current practice nationally.

Supplementary materials: An appendix containing details on orthotic prescription details, prescriptions by diagnosis and rehabilitation scores at admission/discharge is available online or by contacting the lead author.

Author contribution: J.Y. conceived the study and drafted the manuscript. Both authors collected data and approved the finished manuscript.

Funding: No specific funding was received for this research.

Declaration of Conflicting Interests: At the time of conducting the study J.Y. was employed by Opcare, providing an orthotic service within the NHS.

\section{References}

Bowen, A. et al. (2016) National clinical guideline for stroke. Available at: https:// www.strokeaudit.org/SupportFiles/Documents/Guidelines/2016-National-Clinical-Guideline-forStroke-5t-(1).aspx (Accessed: 29 June 2018).

Bowers, R. and Ross, K. (2010) 'Development of a Best Practice Statement on the Use of AnkleFoot Orthoses Following Stroke in Scotland', Prosthetics and Orthotics International, 34(3), pp. 245-253. doi: 10.3109/03093646.2010.486392. 
Elm, E. von et al. (2007) 'Strengthening the reporting of observational studies in epidemiology (STROBE) statement: guidelines for reporting observational studies', BMJ, 335(7624), pp. 806808. doi: 10.1136/bmj.39335.541782.AD.

Hada, T., Momosaki, R. and Abo, M. (2018) 'Impact of orthotic therapy for improving activities of daily living in individuals with spinal cord injury: a retrospective cohort study', Spinal Cord, 56(8), pp. 790-795. doi: 10.1038/s41393-018-0088-9.

International Standards Organization (no date) ISO 8549-1:1989 - Prosthetics and orthotics -Vocabulary -- Part 1: General terms for external limb prostheses and external orthoses. Available at: https://www.iso.org/standard/15800.html?browse=tc (Accessed: 19 June 2018).

McDaid, C. et al. (2017) 'Systematic review of the evidence on orthotic devices for the management of knee instability related to neuromuscular and central nervous system disorders.', BMJ open. BMJ Publishing Group, 7(9), p. e015927. doi: 10.1136/bmjopen-2017-015927.

Momosaki, R. et al. (2015) 'Effects of Ankle-Foot Orthoses on Functional Recovery after Stroke: A Propensity Score Analysis Based on Japan Rehabilitation Database', PLOS ONE, 10(4), p. e0122688. doi: 10.1371/journal.pone.0122688.

Slijper, A., Danielsson, A. and Willén, C. (2012) 'Ambulatory Function and Perception of Confidence in Persons with Stroke with a Custom-Made Hinged versus a Standard Ankle Foot Orthosis.', Rehabilitation research and practice. Hindawi Limited, 2012, p. 206495. doi: 10.1155/2012/206495.

Teasell, R. W. et al. (2001) 'Physical and functional correlations of ankle-foot orthosis use in the rehabilitation of stroke patients', Archives of Physical Medicine and Rehabilitation, 82(8), pp. 10471049. doi: 10.1053/apmr.2001.25078.

The National Institute for Health and Care Excellence (2013) NICE CG162 Stroke rehabilitation in adults. Available at: https://www.nice.org.uk/guidance/cg162/resources/stroke-rehabilitation-inadults-pdf-35109688408261 (Accessed: 19 June 2018).

Turner-Stokes L, et al. (2008) 'Long-term neurological conditions: management at the interface between neurology, rehabilitation and palliative care'. Available at: www.rcplondon.ac.uk (Accessed: 5 Sep 2018).

Turner-Stokes L, et al. (1999) 'The UK FIM+FAM: development and evaluation. Functional Assessment Measure', Clinical Rehabilitation, 13(4), pp. 277-87.

Tyson, S. F. et al. (2017) 'Bespoke versus off-the-shelf ankle-foot orthosis for people with stroke: randomized controlled trial.', Clinical Rehabilitation, pp. 367-376. doi: 10.1177/0269215517728764.

Williams, S., Singer, B. and Lamont, P. (2018) 'Balance and falls in people with Charcot-MarieTooth disease: A cohort survey', International Journal of Therapy and Rehabilitation. MA Healthcare London , 25(1), pp. 6-12. doi: 10.12968/ijtr.2018.25.1.6. 